Japanese Journal of Physiological Psychology and Psychophysiology

Vol. 7, No. 1, 1989. 39-46.

SHORT COMMUNICATION

\title{
EFFECTS OF HEART RATE BIOFEEDBACK TRAINING ON PHYSIOLOGICAL RESPONSES AND THE PROPOSITIONAL STRUCTURE OF FEAR IMAGERY : A CASE REPORT
}

\author{
HIROTA, Akihisa \& HIRAI, Hisashi \\ Sophia University \\ Department of Psychology, Sophia University, \\ 7-1, Kioicho, Chiyoda-ku, Tokyo 102, Japan
}

\begin{abstract}
The present study investigated the effects of biofeedback training to decrease heart rate on psychophysiological responses and the propositional structure of fear imagery. A female student who showed the most physiological responses during fear imageries was selected as the present case-study subject. She was trained to decrease her heart rate according to biofeedback signals during fear imagery for 8 months. After the training, her physiological responses were measured again during fear imageries, where she was asked to control her heart rate without any feedback signals. Finally, questionnaires were administered to examine possible modification in the propositional structure of fear imagery. Results showed that after the training she achieved heart rate control without any feedback signals during fear imageries. Frontal EMG and respiration also showed decrement in post-training. Peripheral skin temperature in post-training, however, decreased more than in pre-training. It is suggested that the response propositions of fear imagery are modified in the direction of relaxation through the training to decrease heart rate during fear imagery.
\end{abstract}

Key words : training to decreases heart rate, physiological responses, fear, imagery, propositional structure of imagery.

It is well known that the fear imagery can evoke certain kinds of psychophysiological responeses (Bauer \& Craighead, 1979 ; Marks \& Huson, 1973 ; Schwartz, Weinberger, \& Singer, 1981 ). Wolpe (1958) hypothesized that a basic assumption for systematic desensitization is that the response to an imagined situation resembles the one of a real situation. Thus, Wolpe treated the patient by using fear imageries. The concept of the theory of imagery, how-

— Received April 9, 1988 ; accepted May 26, 1989 廣田昭久・平井 久 ever, was not yet clearly consolidated in his hypothesis. Therefore, it is difficult to understand what was or what was not modified in his successful treatment using the fear imagery.

Lang (1979, 1984) proposed that physiological responses during emotional imagery were determined by the response propositions included in the propositional structure of the imagery.

Moreover, Lang presumed that the two output variables of emotional imagery were verbal report and physiological response, and 
that the modification of either verbal report or efferent physiological response would feedback to modify both the content and the propositional structure of the image. He developed a proposition oriented training using the above presumption, then showed that the changes of verbal report led to the modification of the structure of the imagery (Lang, Kozak, Miller, Levin, \& McLean, 1980 ; Lang, Levin, Miller, \& Kozak, 1983 ; Lang, Miller, \& Levin, 1983 ). However, Lang did not examine the latter path, that is, the modification of the propositional structure of imagery through a systematic shaping of the physiological output. The present study is an effort to investigate this second path. We tried to modify the structure of fear imagery through a procedure in which one subject was trained to decrease her heart rate during fear imagery.

We hypothesized that the characteristic of the response propositions would be modified, that is, the contents of the response propositions would be modified to set free a more arousing nature. A questionnaire was used to investigate the changes in contents of the response propositions.

It is known that the situation of a snakeexposure or a social-performance generally causes fear. The present study also tried to investigate these two types of fear imagery. Therefore, a subject who was afraid of both types of fearful situations was selected using a snake-fear questionnaire, Snake Anxiety Questionnaire (SNAQ) and a speech-fear questionnaire, Personal Report of Confidence as a Speaker( PRCS ). These two questionnaires were used by Lang and his associates (1983) and were translated into Japanese with some revisions. ${ }^{1)}$

1) We greatly appreciate his kindness to send us much literature including appendices.

\section{Method}

\section{Subjects}

The subject was a female undergraduate student. Her age was 19 years at the beginning of the present study but became 20 years before its completion.

\section{Apparatus}

Inter-heartbeat-interval ( IBI ), frontalis EMG, peripheral skin temperature, and respiration were all recorded. ECG was measured using the standard lead II derivation. The IBI was recorded using a homemade IBI timer with a precision down to $1 \mathrm{~ms}$. The output from the timer was fed into a NEC PC-8801mk II personal computer system which processed IBI data and stored them on a magnetic disk. EMG was recorded from the frontalis muscle group by a pair of $\mathrm{Ag} / \mathrm{AgCl}$ surface electrodes. EMG was amplified and integrated by Autogen 1100 (Autogenic Systems, Inc.), and the results were also stored on the magnetic disk of the PC-8801 after A/D conversion. The peripheral skin temperature was measured by Autogen 1000b (Autogenic Systems, Inc.). A thermistor was attached to the tip of the right middle finger. Respiration was measured by means of a strain gauge transducer attached to a belt (Nihon Kohden MCR-1TA) which was strapped around the chest. The output of the transducer was recorded on a recticorder (Nihon Kohden ).

Tactile feedback was used during the training session. The feedback was in the form of a continuous vibration given to the tip of the left index finger. The frequency of vibration varied with IBI : The longer IBI (heart rate deceleration) produced a decrease in frequency of the feedback vibration, whereas the shorter one ( heart rate acceleration) produced a higher frequency of vibration. The variation of frequency in vibration was contorolled by the PC 8801 . 


\section{Procedure}

The original SNAQ and PRCS were translated into Japanese, then 26 items in each questionnaire were used. In order to examine the response proposition structure, a Response Proposition Questionnaire ( RPQ) was constructed from Anxiety APQ (Autonomic Perception Questionnaire) by Mandler and his associates (1958) with some revisions. Each of the 25 items of RPQ deals with the perception of 7 kinds of bodily reaction. Subjects are asked to rate the degree of would-be reaction on a 5 - point scale $(1=$ Never, $5=$ Always $)$ when in a state of fear. They are instructed to think that they are in a certain fearful situation when they are given RPQ. Therefore, they must at least imagine some situation in which they feel fear.

Thus, RPQ was used in the present study on the assumption that RPQ could find the structure of response proposition in fear imagery which the subject had.

The three questionnaires were administered to 45 male and 61 female undergraduate students. The subjects who showed high scores in both SNAQ and PRCS, listed more bodily reactions and rated high arousal for each item, especially for heart rate items, were asked to come to the laboratory for further examination.

In the first session each subject was asked to write four situations which he or she felt the highest degree of fear for, 4 individual fearful scripts (individual scenes) were constructed later. The subject was seated in a comfortable chair in a dimly-lit room. All elecrtrodes were attached to the subject, and he or she was instructed to sit quietly with his or her eyes closed for 30 minutes. One more adaptation session was given to the subject in the second day. On this session he or she was also instructed to ramain quietly sitted.

On the third day, the subject took the pre-training imagery test. Psychophysiological responses were measured during some kind of imagery. He or she was asked to imagine ten scenes : 4 neutral, 2 snake, 2 social performance, and 2 individual ones. All scenes were constructed only of stimulus propositions. The first two scenes were neutral and were excluded from our analysis, because we knew from the pilot experiment that physiological responses in the first scenes had always excessively large variability. All scripts were pre-recorded on a cassette recorder and presented through a speaker.

One script was used in each trial which was divided into four time periods : a $20 \mathrm{~s}$ rest period, a $50 \mathrm{~s}$ listen period during which a script was presented and the subject imagined it, a $20 \mathrm{~s}$ image period in which the subject continued to imagine the scene after the termination of the script, and a $20 \mathrm{~s}$ recover period after stopping imagery.

The female subject who showed the most physiological changes, especially heart rate acceleration, in the pre-training imagery test (see Fig. 2) was selected to continue the following training with her consent.

She received a feedback-and-neutral-imagery training followed by a feedback-and-fearimagery training. She took two sessions per week on the average. Both trainings contained 12 trials. In the first six trials, the subject was asked to try to decrease her heart rate using a tactile feedback. Each trial was divided into two time periods : a $20 \mathrm{~s}$ baseline period, and a $70 \mathrm{~s}$ training period during which the subject tried to accomplish the imposed task. On the next six trials (the feedback-and-imagery trials), she was told to form an image of the script presented to her and simultaneously to try to decrease her heart rate by feedback. Each trial was divided into two parts : The first $20 \mathrm{~s}$ were spent to measure the baselines of various physiological responses ; then during the next $70 \mathrm{~s}$ she tried to do the imposed task. 
Two neutral scripts were alternately used in the feedback-and-neutral-imagery training, wereas in the feedback-and-fear-imagery training, 6 fear scripts were presented : 2 snake, 2 social performance, and 2 individual scenes. All scripts used in both trainings were different from the ones used in the pre-training imagery test.

The feedback-and-neutral-imagery training was continued for a period of one month. The training was terminated on session 13 .

A feedback-and-fear-imagery training was then performed on her. This training was continued for 8 months. The tactile feedback was no more given to the subject from session 23 on through session 29 , because she reported that the feedback stimulus appeared to be interfering with the control of her heart rate. Her performance in controling her heart rate, however, did not improve by the elimination of the feedback, so that it was resumed from session 30 on. The feedback-and-fear-imagery training was terminated on session 37 .

Next, she participated in the post-training imagery test. She imagined the same ten scenes which were used in the pre-training imagery test. And she was instructed to control her heart rate without the assistance of feedback.
Sixteen days later, she took a test of generalization. She was again told to decrease her heart rate under the no-feedback condition during imagery. Seven new scenes ( 3 neutral, 2 snake, and 2 social performance) which were never used before were presented to her with the same procedure used in pre- or post-training imagery tests. The first neutral scene was excluded from analysis.

Finally, SNAQ, PRCS, and RPQ were again administered to her. The entire experiment continued for nine months.

\section{Results}

For the feedback-and-imagery trials, the change scores which were obtained by subtracting the mean IBI for the baseline period from the mean IBI for the training period are presented in Fig. 1. The IBI gradually became longer through the sessions, though immediately after the introduction of the fear scenes, the IBI became remarkably shorter. In fact, her IBI reached minus 207 during an individual scene. Finally, she could accomplish deceleration of her heart rate (mean +27.5$)$ in every fear scene, therefore, the training terminated on session 37, though IBI temporarily became shorter in and after the no-feedback sessions.

Frontalis EMG were decreased in almost

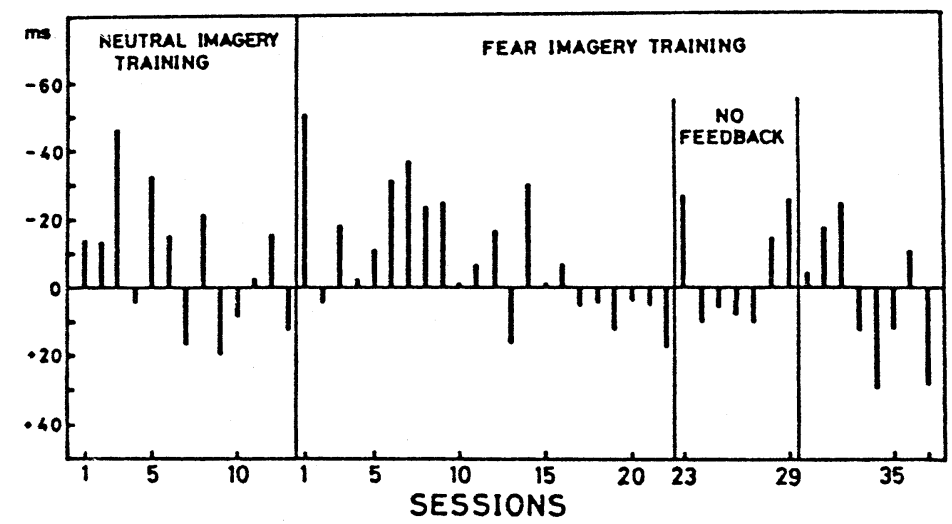

Fig. 1 Changes in mean inter-heartbeat-interval(IBI) from the baseline period in the feedback-and-neutral-imagery training and the feedback-and-fear-imagery trainings. 
every training session. The change score was -.13 on session 1 of the feedback-and-fearimagery training, then the values gradually decreased and the value of final session 37 was -.52 .

Peripheral skin temperature fluctuated through sessions. The values, however, decreased to reach under the baseline level in almost every session.

Respiration increased over the baseline level $(+2.31)$ immediately after the fear imagery, then it gradually decreased to reach below the baseline $(-.97$ on session 37$)$ through the sessions.

For the purpose of analysing the data of the pre- and post-training imagery tests, change scores were created using average values for each period by subtracting the rest period values from the listen, image, and recover period scores. These change scores were then averaged over two trials of each neutral, snake, social performance, and individual scene for each period. The changes of the four physiological responses are presented in Fig. 2. The $\mathrm{L}, \mathrm{I}$, and $\mathrm{R}$ on the horizontal axis indicate the listen, image, and recover periods, respectively.
The IBIs in the post-training were obviously lower than the corresponding IBIs of pretraining in every scene. In the most extreme case, the difference between the pre- and post-training reached as much as about $200 \mathrm{~ms}$ in the image period of the social performance scene.

In respiration, the change scores of the post-training were also obviously lower than those of the pre-training in each scene. During post-training, moreover, respiration decreased even under the level of rest period.

After the training, the change scores of EMG were kept under about $.4 \mu \mathrm{V}$ in every scene. In the snake and individual scenes, increases in post-training were suppressed much better in comparison with those of pre-training. On the contrary, the change score of pre-training was lower than the one of post-training in social performance scenes. The probable reason for this result was that a trial of the social performance scene is the pretraining imagery test started when EMG was not stable and did not yet sufficiently recover.

The peripheral temperature decreased below the level of the rest period in every scene.

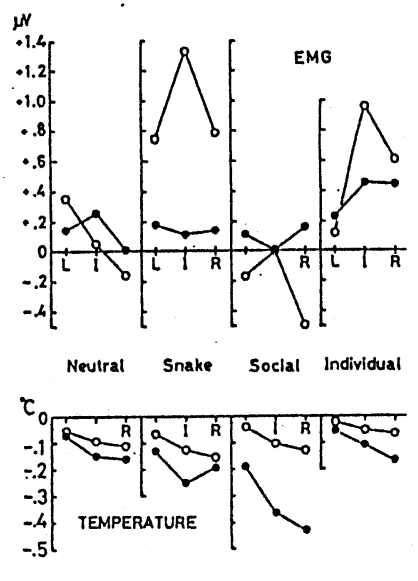

Fig. 2 Mean changes in IBI, frontal EMG, respiration, and fingertip temperature from the preceding rest period in the listen (L), image (I), and recover (R) periods. Results are shown for pre-training and post-training imagery tests during neutral, snake, social performance, and individual scenes. 
Also, the peripheral temperature in the posttraining was always lower than the one in the pre-training.

Figure 3 shows the results of generalization. For the IBI, the acceleration in the social performance scene was about the same as the one of the post-training imagery test. As in the post-training imagery test, both respiration and peripheral temperature were decreased below the level of the rest period. The frontalis EMG did not show remarkable changes in any scenes.

In order to examine the modification of the propositional structure in fear imagery, the change scores of SNAQ and PRCS were determined by subtracting the scores for pre-training from the score which was obtained after training. Also, change scores of RPQ were computed likewise for each item. Concerning SNAQ and PRCS, their change scores were +1 and -1 , respectively. Thus, in SNAQ and PRCS, no marked difference was observed between the pre- and the post-trainings. In regard to the direction of change in rating of each item of RPQ, eight items were modified in the direction of relaxation, whereas 3 items were modified in the direction of arousal. The average change of ratings for the former 8 items was -1.0 ( ranged from -0.5 to -2 ), on the other hand, the mean change of the latter 3 items was +0.67 ( ranged from +0.5 to +1.0 ).

\section{Discussion}

It might be that the results in the training session reflected a simple adaptation. However, the subject was able to inhibit the acceleration of her heart rate even in the post-training imagery test and the test of generalization where she was given no feedback of heart rate. Moreover, the seven scenes presented in the test of generalization were never used before and then they were novel stimuli to her. Therefore, it is considered that the subject acquired the ability to control her heart rate through the training sessions.

We noted that frontalis EMG also decreased when the subject tried to decrease her heart rate. Additionally, it was found that the heart rate deceleration was accompanied with a decrease in respiration. The EMG biofeedback has so far been considered to be the most effective method of relaxation, however, the present results suggest that a training to decrease heart rate is also effective in the
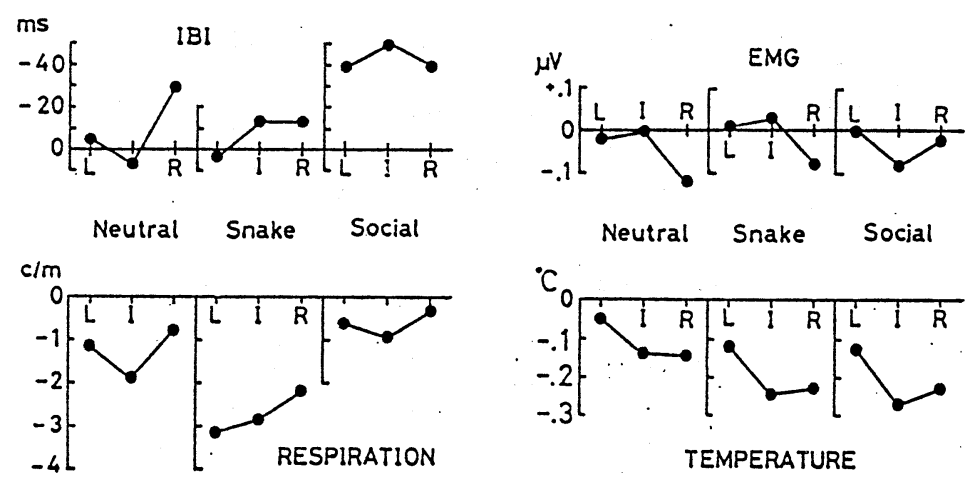

Fig. 3 Mean changes in IBI, frontal EMG, respiration, and fingertip temperature from the preceding rest period in the listen (L), image (I), and recover (R) periods. Results are shown for the test of generalization during neutral, snake, and social performance scenes. 
acquisition of relaxation.

The results in the post-training imagery test and the test of generalization showed that the subject was able to inhibit the acceleration of her heart rate in the fear scenes. This subject seemed to gain control over her heart rate in a fearful situation without the assistance of feedback.

EMG and respiration were decreased with the deceleration in heart rate. It is considered that the reduction in heart rate, muscle, and respiration activities reflect a relaxation. On the other hand, peripheral skin temperature is considered to be an index of the sympathetic activation. Therefore, the peripheral temperature must be increased if the sympathetic activation is reduced, that is, in the relaxation. The present results, however, showed that the peripheral temperature was not increased, rather, it was always decreased below the baseline level. Moreover, the amount of temperature reduction was larger in the posttraining than the one in the pre-training. The decreased peripheral temperature might reflect a psychological load to inhibit the acceleration of heart rate in fearful situations.

Regarding the modification of the propositional structure of the fear imagery, it was found that the situation in which the subject felt some fear was not modified because the scores for SNAQ and PRCS in the post-training were not greatly different from those in the post-training. On the other hand, the number of items modified in the direction of relaxation was larger than the one of items modified in the direction of arousal. The results suggest that the response propositions of fear imagery are altered in the direction of relaxation through a training meant to decrease heart rate during fear imagery. It is suggested that fear itself might be reduced by the physiological modification in this case.

It the present study, it is suggested that the propositional structure of fear was modified. The mechanisms for the modification, however, were not investigated. Foa and Kozak (1986) described that interoceptive information about the absence of physiological arousal is generated when physiological responses decrease during confrontation with fearful situations, and that the information is available for encoding as response propositions that are inconsistent with those of the structure and then weakening the preexisting links between stimulus and response elements. According to their hypothesis, in the present study the new information was encoded as response proposition while the subject controlled the decrease of heart rate during fear imagery in the training sessions, and that the links between stimulus and response propositions were modified at that moment.

\section{References}

Bauer, R. M., \& Craighead, W. E. 1979 Psychophysiological responses to the imagination of fearful and neutral situations : The effects of imagery instructions. Behav. Ther., 10, $389-403$.

Foa, E. B., \& Kozak, M. J. 1986 Emotional processing of fear : Exposure to corrective information. Psychol. Bull., 99, 20-35.

Lang, P. J. 1979 A bio-informational theory of emotional imagery. Psychophysiology, 16, $495-512$.

Lang, P. J. 1984 Cognition in emotion : Concept and action. In C. E. Izard, J. Kagan, \& R. Zajonc (Eds.), Emotions, Cognition, and Behavior New York : Cambridge University Press. Pp. 192-226.

Lang, P. J., Kozak, M. J., Miller, G. A., Levin, D. N., \& McLean, A. J. 1980 Emotional imagery : Conceptual structure and pattern of somatovisceral responses. Psychophysiology, 17, 179-192.

Lang, P. J., Levin, D. N., Miller, G. A., 
\& Kozak, M. J. 1983 Fear behavior, fear imagery, and the psychophysiology of emotion : The problem of affective response integration. J. Abnorm. Psychol., 92, 276306.

Lang, P. J., Miller, G. A., \& Levin, D. N. 1983 Anxiety and fear : Central processing and peripheral physiology. In R. J. Davidson, G. E. Schwartz, \& D. Shapiro (Eds.), Consciousness and Self-regulation : Advances in Research and Theory : Vol. 3. New York : Plenum Press. Pp. 123-151.

Mandler, G., Mandler, J. M., \& Uviller, E. T. 1958 Autonomic feedback : The perception of autonomic activity. J. Abnorm. Soc. Psychol., 56, 367-373.

Marks, I. M., \& Huson, J. 1973 Physiological aspects of neutral and phobic imagery : Further observation. Brit. J. Psychiat., 122, $567-572$.

Schwartz, G. E., Weinberger, D. A., \& Singer, J. A., 1981 Cardiovascular differentiation of happiness, sadness, anger, and fear following imagery and exercise. Psychosom. Med., 43, $343-364$.

Wolpe, J. 1958 Psychotherapy by Reciprocal Inhibition. Stanford, Calif. : Stanford University Press.

正 誤 表 第 6 巻第 1 号

(市井・根建 論文)

$\begin{array}{ccccc}\text { ページ } & \text { 列 } & \text { 行 } & \text { 誤 } & \text { 正 } \\ 25 & \text { 右 } & \uparrow 7 & \text { Psychophysiology } & J p n . \text { Psychol. Res. }\end{array}$

